\section{New evidence on atenolol in hypertension}

\section{EBM}

A new systematic review by Carlberg and colleagues has revealed that atenolol, one of the most common $\beta$-blockers, might not be appropriate as a first-line treatment for hypertension.

In a meta-analysis of four randomized, controlled trials comparing the drug with placebo (or no treatment) in 6,825 patients, there were no overall differences in the risk of all-cause mortality, cardiovascular mortality or myocardial infarction during a mean follow-up of 4.6 years. Patients receiving atenolol tended to have a lower risk of stroke than patients on placebo; one study showed a $43 \%$ reduction in stroke risk, but most patients in that study were treated with more than one antihypertensive drug. All the studies showed a marked reduction in blood pressure using atenolol.

The authors also carried out a meta-analysis of five trials (17,671 patients) comparing atenolol with other antihypertensive agents. Although atenolol showed similar efficacy to the other drugs in terms of blood-pressure lowering, there was significantly higher mortality in the atenolol group during the mean follow-up of 4.6 years. Importantly, cardiovascular mortality was higher in the atenolol group, as was the risk of stroke.

Carlberg et al. conclude that the suitability of atenolol as an antihypertensive agent is now uncertain. Its role as a reference drug in trials of antihypertensive agents might no longer be appropriate.

Original article Carlberg B et al. (2004) Atenolol in hypertension: is it a wise choice? Lancet 364: 1684-1689

\section{ACE inhibition shows no benefit in the PEACE trial}

Angiotensin-converting-enzyme (ACE) inhibitors are well established in the prevention of cardiovascular events in patients with heart failure or left-ventricular systolic dysfunction. In addition, these agents have improved outcomes in high-risk patients with vascular disease and normal left-ventricular function. The Prevention of Events with Angiotensin Converting Enzyme Inhibition (PEACE) trial was designed to investigate whether low-risk patients with coronary artery disease would also benefit from adding an ACE inhibitor to their usual therapy.

Participants were randomized to $4 \mathrm{mg}$ trandolapril daily $(n=4,158)$ or placebo $(n=4,132)$. All had stable coronary artery disease and preserved left-ventricular function, and most were receiving lipid-lowering drugs or had undergone coronary revascularization. The primary endpoint of the study was a composite of death from cardiovascular causes, myocardial infarction or coronary revascularization.

During a median follow-up of 4.8 years, the incidence of the primary endpoint was similar in the trandolapril and placebo groups $(21.9 \%$ vs $22.5 \%, P=0.43)$. Prespecified secondary endpoints also occurred at similar rates in the two treatment groups. Cough and syncope were recorded significantly more frequently in the trandolapril group.

The patients in this study had a low rate of cardiovascular events relative to rates in earlier ACE inhibitor trials, but trandolapril therapy failed to reduce this further. It may be appropriate, however, to prescribe ACE inhibitors to these patients under certain circumstances, for example patients with diabetes or hypertension.

Original article The PEACE Trial Investigators (2004) Angiotensin-converting-enzyme inhibition in stable coronary artery disease. N Engl J Med 351: 2058-2068

\section{A-HeFT trial: positive results}

In the first Vasodilator Heart Failure Trial (V-HeFT), the combination of the antioxidant hydralazine with isosorbide dinitrate-which stimulates nitric oxide signaling-was beneficial in patients with mild to severe heart failure. Since then, subgroup analysis of previous trial data has indicated that black patients respond particularly well to this drug combination. The African-American Heart Failure Trial (A-HeFT) studied the efficacy of a fixed dose of isosorbide dinitrate and hydralazine in black patients with advanced heart failure.

A total of 1,050 participants were randomized to active treatment or placebo in addition to their standard therapy (including ACE inhibitors, angiotensin-receptor blockers, $\beta$-blockers and spironolactone). The trial was terminated early because of a striking difference in the 